\title{
High frequency electromagnetic detection by nonlinear conduction modulation in graphene nanowire diodes
}

\author{
M. Winters, ${ }^{1}$ M. Thorsell, ${ }^{1}$ W. Strupiński, ${ }^{2}$ and N. Rorsman ${ }^{1}$ \\ ${ }^{1}$ Department of Microtechnology and Nanoscience, Chalmers University of Technology, Kemivägen 9 , \\ 412-96 Göteborg, Sweden \\ ${ }^{2}$ Institute of Electronic Materials Technology, Wóczyńska 133, 01-919 Warsaw, Poland
}

(Received 20 July 2015; accepted 29 September 2015; published online 8 October 2015)

\begin{abstract}
We present graphene nanowires implemented as dispersion free self switched microwave diode detectors. The microwave properties of the detectors are investigated using vector corrected large signal measurements in order to determine the detector responsivity and noise equivalent power (NEP) as a function of frequency, input power, and device geometry. We identify two distinct conductance nonlinearities which generate detector responsivity: an edge effect nonlinearity near zero bias due to lateral gating of the nanowire structures, and a velocity saturation nonlinearity which generates current compression at high power levels. The scaling study shows that detector responsivity obeys an exponential scaling law with respect to nanowire width, and a peak responsivity (NEP) of $250 \mathrm{~V} / \mathrm{W}(50 \mathrm{pW} / \sqrt{\mathrm{Hz}})$ is observed in detectors of the smallest width. The results are promising as the devices exhibit responsivities which are comparable to state of the art self switched detectors in semiconductor technologies. C 2015 AIP Publishing LLC.
\end{abstract}

[http://dx.doi.org/10.1063/1.4932970]

The detection of high frequency microwave and $\mathrm{THz}$ radiation is of integral importance to a diverse range of disciplines in the physical sciences, and efficient detectors realized in graphene may prove to be of relevance owing to the unique electronic properties of the material. ${ }^{1,2}$ Self switched devices (SSDs) offer a unique alternative to high frequency detection as broken conduction symmetry is achieved via lateral gating. ${ }^{3,4}$ Several studies have been presented in which laterally gated nanowires patterned in semiconducting heterostructures demonstrate asymmetric current/voltage (IV) characteristics thereby enabling high frequency detection at zero bias. ${ }^{5-8}$ The choice of material in these experiments is motivated by the high mobility two dimensional electron gas which forms at the heterostructure interface. This suggests that efficient high frequency detection in graphene nanowire diodes (GNDs) is feasible to similar effect. ${ }^{9-11}$ As monolayer and bilayer graphene are semi-metallic materials exhibiting ambipolar transport, the nonlinearity which facilitates detection is of a different character than that seen in semiconducting nanowire diodes.

Nanowire structures are formed by etching narrow trenches into an electrically isolated graphene mesa. The high frequency GND detector consists of a linear array of GNDs etched into a single mesa which is bridged by a coplanar waveguide (see Fig. 1(a)). The operation of the GND is attributed to a geometric effect, whereby the outlying mesa laterally gates the nanowire when a drain bias $\left(v_{d}\right)$ is applied across the junction (Figs. 1(b) and 1(c)). The responsivity in GNDs is strongly controlled by the channel width $(w)$, channel length $(l)$, and the width of the isolating trenches $\left(w_{0}\right)$ indicated in Fig. 1(c). In particular, the scaling of $w$ is of relevance as it lends insight into the physics of electron transport within the graphene channel. As $w$ decreases, lateral gating becomes more effective, resulting in a stronger nonlinearity and enhanced responsivity. In this work, the nonlinear IV characteristic in GND detectors of various nanowire widths $(100,70,50$, and $30 \mathrm{~nm})$ is connected to trends in responsivity and noise equivalent power (NEP). The GNDs are fabricated via electron beam lithography (EBL) in epitaxial graphene grown by chemical vapor deposition (CVD) on semi-insulating $4 \mathrm{H}-\mathrm{SiC}$ substrates. ${ }^{12}$ After growth, the monolayer graphene is intercalated with Hydrogen resulting in quasi-free standing bilayer graphene with enhanced carrier mobility. ${ }^{13-16}$ Monolayer and bilayer graphene nanowires transition to a semiconducting state with decreasing width due to the lateral confinement of carriers and edge disorder induced Anderson localization. ${ }^{17-21}$ The resulting energy gap $\left(\epsilon_{g}\right)$ becomes relevant on the smallest width scales probed in this study and may contribute to enhanced responsivity in the $30 \mathrm{~nm}$ devices.

DC measurements are performed using a semiconductor parameter analyzer and the results are summarized in Fig. 2(a). In narrow structures, a charge neutrality feature is observed in the conductance near $v_{d}=0$, which is consistent with the lateral gating effect depicted in Fig. 1(b). The charge neutrality feature is gradually occluded with increasing $w$. In order to understand the effect of lateral gating on the zero bias nonlinearity, a one dimensional transport model may be applied to the nanowire. When biased, the electric field couples capacitively from the outlying mesa to the nanowire via the substrate. The GND structure may be modeled as a capacitive divider as shown in Fig. 1(c). $C_{s}$ $=\varepsilon_{0} \kappa / w_{0}$ represents the substrate capacitance, where $\varepsilon_{0}$ is the vacuum permittivity, and $\kappa$ is the relative static permittivity. $\kappa$ may be estimated as the mean of the substrate permittivity and the vacuum permittivity $\kappa=\left(\kappa_{s}+1\right) / 2$. $C_{q}=e^{2} \rho(\epsilon)$ is the quantum capacitance in graphene. The quantity $\rho(\epsilon)$ in $C_{q}$ represents the density of states in graphene as a function of chemical potential $(\epsilon) .^{22-24}$ The quasiFermi potential $(v)$ along the length of the nanowire may be 


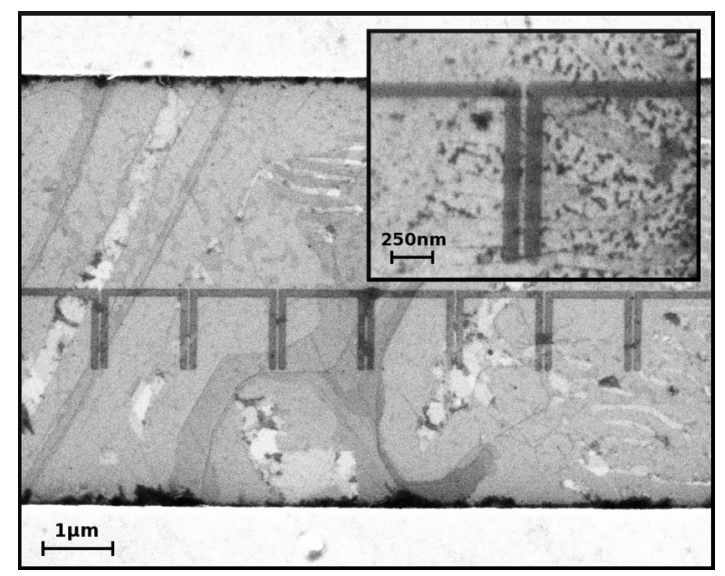

(a)

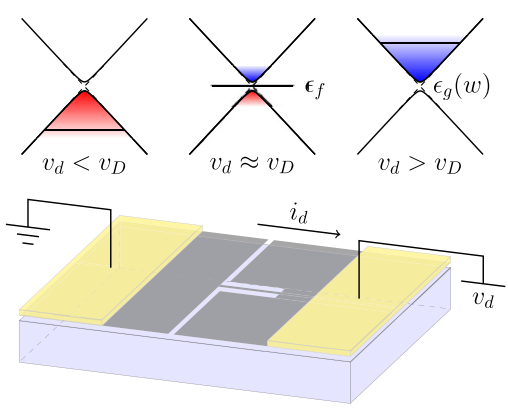

(b)

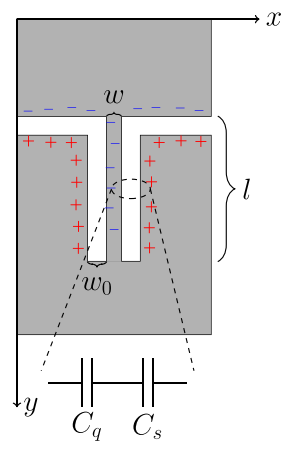

(c)
FIG. 1. (a) A scanning electron microscope (SEM) image of an RF detector consisting of several parallel $30 \mathrm{~nm}$ GND channels. The spacing between channel regions is $1 \mu \mathrm{m}$. (Inset): A SEM micrograph of a single GND channel with high edge acuity. The channel dimensions are $w=30 \mathrm{~nm}$, $w_{0}=100 \mathrm{~nm}$, and $l=1.1 \mu \mathrm{m}$. (b) The topology of a GND showing the orientation of the source/drain contacts, drain bias $\left(v_{d}\right)$ and current flow $\left(i_{d}\right)$ in the GND. Applying a drain bias moves the Fermi level $\left(\epsilon_{f}\right)$ within the nanowire and introduces nonlinearity into the $i_{d}\left(v_{d}\right)$ characteristic. In graphene nanowires, a narrow energy gap opens in the Dirac dispersion with decreasing width $\epsilon_{g}(w)$. (c) Some critical dimensions of the GND structure, including the channel length $(l)$, the channel width $(w)$, and isolation width $\left(w_{0}\right)$. The channel couples capacitively to an applied drain bias via the substrate capacitance $\left(C_{s}\right)$ and quantum capacitance $\left(C_{q}\right)$. Charges illustrate a forward bias condition $v_{d}>v_{D}$.

found by capacitive division $\partial_{v} v_{g}=\left(C_{s}+C_{q}\right) / C_{s}$. Here, the outlying mesa region is imagined as decoupled from the drain and at gate voltage $v_{g}$. The ideal nonlinear current $\left(i_{d}^{n l}\right)$ may be estimated by performing a Sah-Pao style integral over the length of the nanowire, taking the zero temperature approximation, and equating $v_{g}=v_{d}$ (see the supplementary material ${ }^{25}$ ). This results in a current density which varies quadratically with drain bias

$$
i_{d}^{n l} \approx \mu_{e f f} C_{s}(w / l)\left|v_{d}-v_{D}\right| v_{d} .
$$

Here, $\mu_{\text {eff }}$ and $v_{D}$ represent the effective channel mobility and the Dirac voltage, respectively. For $v_{d}>v_{D}$, the channel exhibits electron conduction and $\mu_{\text {eff }}=\mu_{n}$. For $v_{d}<v_{D}$, the channel exhibits hole conduction and $\mu_{\text {eff }}=\mu_{p}$. In the case of charge neutrality $v_{d} \approx v_{D}$ and nonzero temperature, carrier transport becomes ambipolar (see Fig. 1(b)) such that the discontinuous current zero in Eq. (1) manifests as a continuous current minimum. As $\mu_{n} \neq \mu_{p}$, the IV characteristic may exhibit weak asymmetry about $v_{D}$.

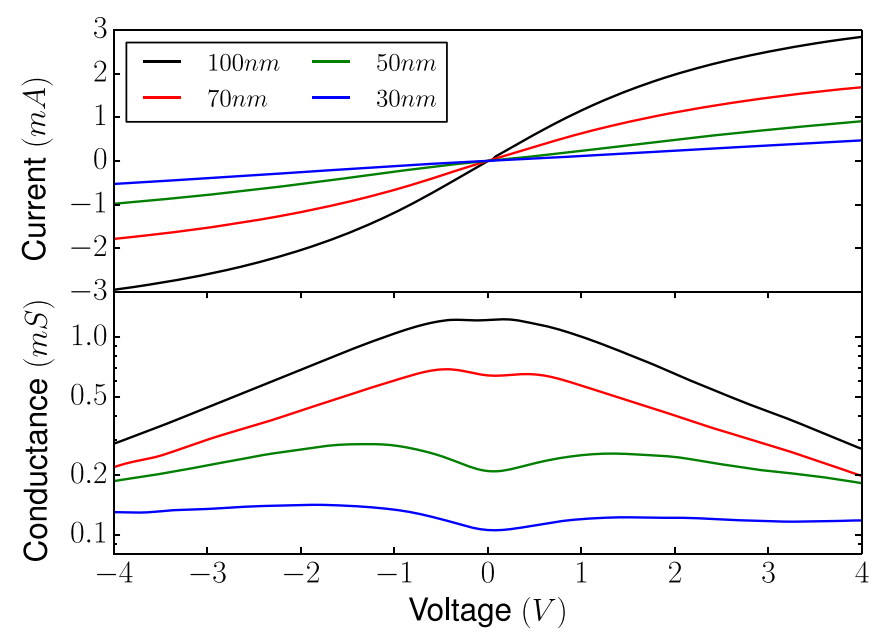

(a)

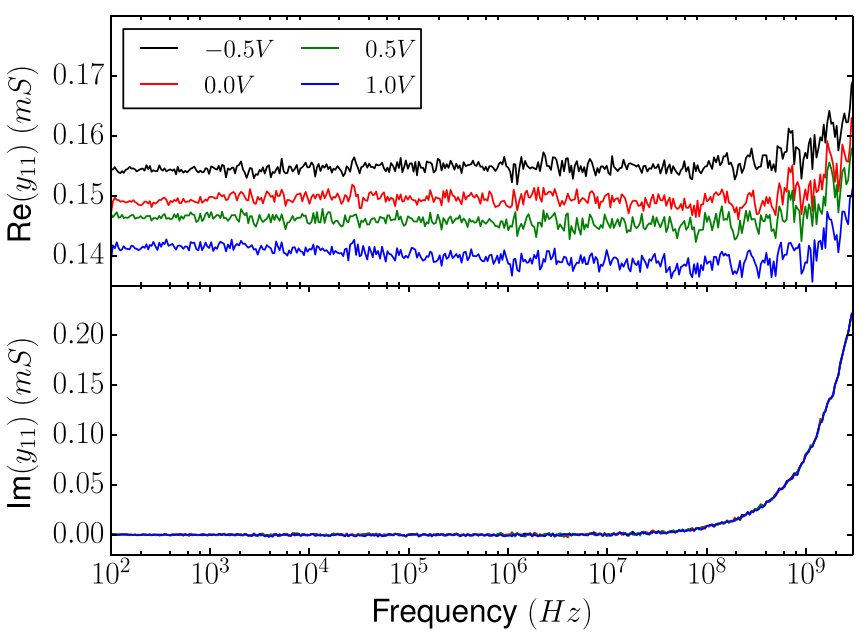

(b)

FIG. 2. (a) DC measurements of two-terminal GND devices of various widths. Note the charge neutrality feature near zero bias for the narrow GNDs. The calculated differential conductance $g=\partial_{v} i$ is also shown (b). The real and imaginary part of $y_{11}$ as calculated from low frequency S-parameter measurements performed at several drain biases. $\operatorname{Re}\left(y_{11}\right)$ is flat with frequency, suggesting an absence of dispersive and trap related effects. $\operatorname{Im}\left(y_{11}\right)$ is identical for all biases and shows the effect of pad capacitance $C_{\text {pad }}$.

In this analysis, the chemical potential is assumed to be constant across the width of the nanowire. In reality, charge carriers move towards the edges of the nanowire and screen the electric field induced by lateral gating. The distance which the field penetrates into the nanowire is characterized by the Thomas-Fermi screening length $r_{s}=\left[\alpha k_{f}\right]^{-1}$. Here, $\alpha=e^{2} / \kappa \hbar v_{f} \approx 2.2 / \kappa$ is the fine structure constant in graphene, and $k_{f}=\sqrt{4 \pi n_{s} / g_{s} g_{v}}$ is the Fermi-wavevector. ${ }^{26}$ For $4 \mathrm{H}-\mathrm{SiC}, \kappa=\left(\kappa_{\|}+1\right) / 2 \approx 5.5$ which yields $\alpha \approx 0.18 .{ }^{27}$ The field penetrates the nanowire equally on both sides such that strong nonlinearity is expected when the width of the nanowire is of order $w_{c}=2 r_{s}$. Near zero bias, the carrier density in H-intercalated graphene is $n \approx 1 \times 10^{13} \mathrm{~cm}^{-2}$ such that $w_{c} \approx 20 \mathrm{~nm}$. It is important to note that this analysis describes the case of a monolayer and does not account for nonlinear polarization effects. A similar argument for bilayer material yields a value $w_{c} \approx 26 \mathrm{~nm}$. 


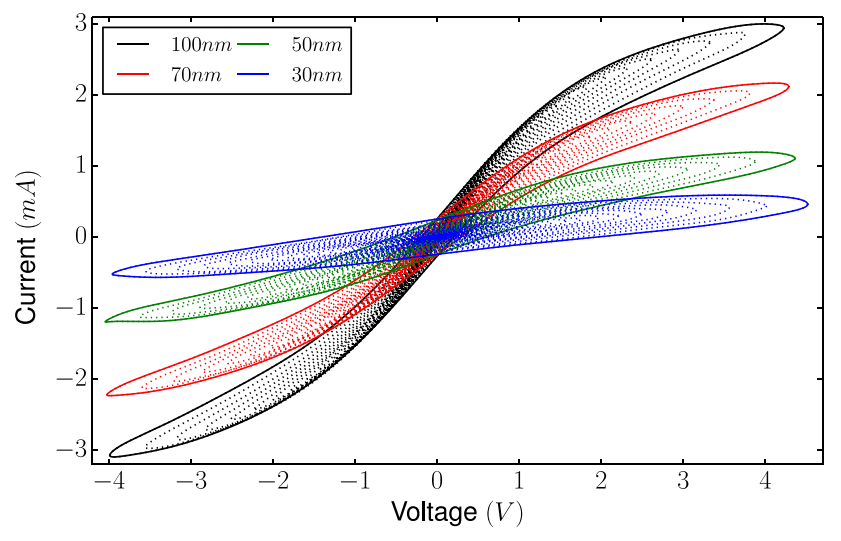

(a)

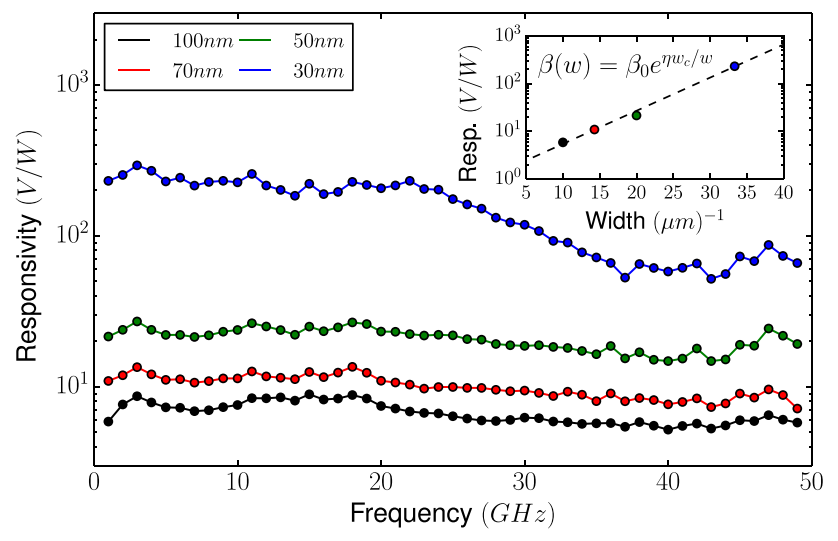

(c)

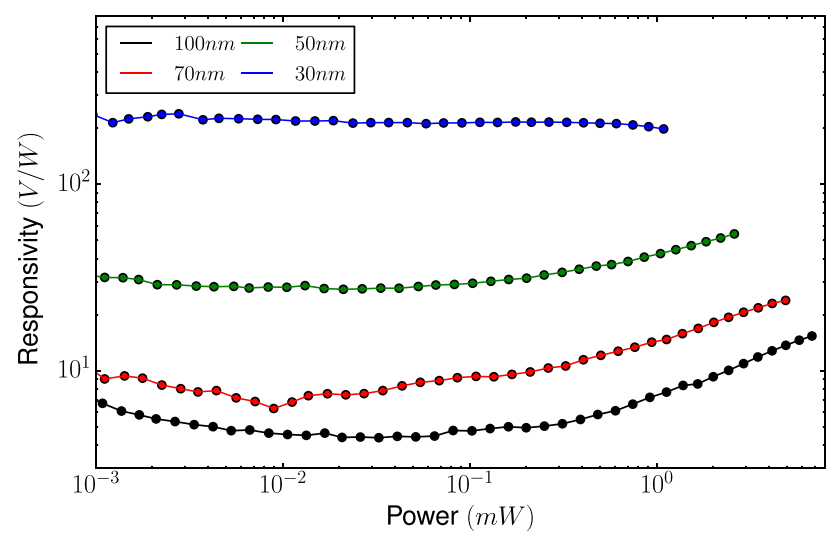

(b)

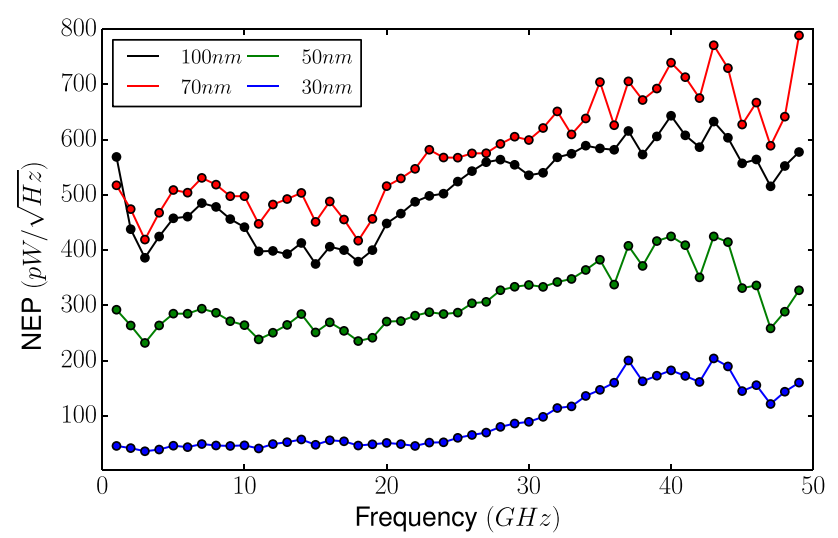

(d)

FIG. 3. (a) IV waveforms of GND detectors of various widths demonstrating resistive scaling with increasing input power. The $1 \mathrm{GHz}$ waveforms extracted from large signal measurements closely resemble the DC results shown in Fig. 2(a). (b) DC responsivity plotted as a function of delivered power for the GNDs shown in Fig. 3(a). (c) DC responsivity plotted as a function of frequency for a $0 \mathrm{dBm}$ excitation from $1 \mathrm{GHz}$ to $49 \mathrm{GHz}$. (Inset) Responsivity scaling with GND width at $1 \mathrm{GHz}$. The data reveal an exponential scaling law (dashed). (d) NEP at $k_{b} T=26 \mathrm{meV}$ as calculated for the GND structures shown in Fig. 3(c).

The conductance minimum observed at the charge neutrality point is expected to be strongly enhanced when the energy gap in the graphene nanowires is of order $k_{b} T$. In Ref. 18, the energy gap is shown to scale inversely with nanowire width $\epsilon_{g}(w)=a\left(w-w^{*}\right)^{-1}$, where $a=200 \mathrm{meV}$ $\mathrm{nm}$ and $w^{*}=16 \mathrm{~nm}$ are empirical constants. For the width scales considered in this study, the energy gap is expected to range from $2 \mathrm{meV}$ for the $100 \mathrm{~nm}$ devices to $14 \mathrm{meV}$ for the $30 \mathrm{~nm}$ devices. The energy gap is especially relevant in the case of narrow devices as it introduces a strong temperature dependence into the conductance minimum at zero bias. ${ }^{18,21}$ In this work, $k_{b} T>\epsilon_{g}$ such that the energy gap effect is sufficiently suppressed due to thermalisation of carriers for all devices with the possible exception of the $30 \mathrm{~nm}$ devices in which an enhanced zero bias nonlinearity is observed.

When $w$ is more than a few $w_{c}$, lateral gating only produces an edge effect and the core of the nanowire behaves as a normal linear conductor $\left(g_{0}\right)$. This interpretation of device operation is supported by the work of Panchal et al. in which carrier transport in laterally gated graphene devices is investigated using scanning probe techniques. ${ }^{28}$ In Ref. 28 , p-type edge conduction is observed in n-type bulk graphene as a consequence of lateral gating. Additionally, the width of the edge conducting channels is estimated at $60-125 \mathrm{~nm}$ which is of the order of a few $w_{c}$ in their material. Thus, at low bias, the GND IV characteristics observed in Fig. 2 may be modeled by a linear bulk conductance and a nonlinear edge conductance in parallel such that $i_{d}=\left[g\left(v_{d}\right)+g_{0}\right] v_{d}$. The nonlinear component $i_{d}^{n l}=g\left(v_{d}\right) v_{d}$ is approximated via Eq. (1).

As $v_{d}$ increases, the conductivity begins to decrease as a consequence of velocity saturation. As the nanowires are highly resistive relative to the ohmic contacts and access resistances, the majority of the voltage drop due to an applied $v_{d}$ occurs over the length of the wire $(l=1.1 \mu \mathrm{m})$. The onset of velocity saturation in $\mathrm{H}$-intercalated graphene occurs at an electric field of $\mathcal{E}_{c} \approx 20 \mathrm{kV} / \mathrm{cm}^{29}$ In the fabricated devices, $\mathcal{E}_{c}$ is reached at approximately $2.2 \mathrm{~V}$ and coincides with the observed current compression and decrease in conductivity at high bias. In graphene, velocity saturation occurs when carriers have sufficient energy to scatter via emission of polar optical phonons in the substrate. ${ }^{29-31}$

Low-frequency bias dependent S-parameter measurements are performed in order to investigate trapping effects in the GNDs (Fig. 2(b)). The small signal conductance $\operatorname{Re}\left(y_{11}\right)$ is observed to be nearly constant with frequency dispersion free operation. Similarly, the small signal susceptance $\operatorname{Im}\left(y_{11}\right)$ reflects a pure capacitance and is attributed to 
the capacitance of the contact pads $\left(C_{\text {pad }}\right)$. The behavior of $y_{11}$ in addition to the absence of DC hysteresis suggests an overall absence of trapping effects in the device which would otherwise generate $1 / f$ noise. Thus, the dominant noise contribution in GND devices is Johnson-Nyquist noise. ${ }^{32}$ The NEP is defined via the ratio of RMS noise voltage and responsivity $\sqrt{\left\langle v_{n}^{2}\right\rangle} / \beta$ and is thus approximated by

$$
N E P=\sqrt{4 k_{b} T r_{d}} / \beta .
$$

Here, $k_{b} T$ is the thermal energy, $r_{d}$ is the zero-bias differential resistance of the entire device. For a given responsivity, the NEP may be reduced by simply increasing the number of parallel GND channels. Increasing $\mu_{n, p}$ has a similar effect of reducing $r_{d}$ such that NEP also improves with material quality. The lack of dispersion may be attributed to the fact that the devices as fabricated are unpassivated, thus precluding interface traps and associated material degradation resulting from dielectric deposition. ${ }^{24}$

Responsivity in GND detectors may be interpreted as a consequence of two interacting nonlinearities: the charge neutrality nonlinearity near zero bias and the saturation nonlinearity at high bias (Fig. 2(a)). Responsivity measurements are carried out using a large signal network analyzer (LSNA). The high impedance of the GND detectors needs to be accounted for, hence vector corrected power measurements are used in order to correctly determine the detector responsivity $\left(\beta=v_{D C} / P_{i n}\right)$. The LSNA enables vector corrected measurements with amplitude and phase information at the fundamental frequency as well as at higher order harmonics. ${ }^{33}$ The delivered RF power $\left(P_{i n}\right)$ to the GND is measured at $1 \mathrm{GHz}$ by the LSNA, and a voltage meter is used to measure the DC voltage across the diode generated by the nonlinearity $\left(v_{D C}\right)$. The resulting vector corrected responsivity for various input powers is shown in Fig. 3(b). The nonlinear IV waveforms at the GND terminal are then directly reconstructed via inverse Fourier Transform of the amplitude and phase data obtained from the LSNA measurements such that details about device operation can be observed. The IV waveforms versus input RF power are shown in Fig. 3(a), illustrating the approximately symmetrical characteristics of the GND. Current compression is observed in the waveforms as a consequence of velocity saturation, in line with the DC measurements in Fig. 2(a). The agreement between the $1 \mathrm{GHz}$ waveforms of (Fig. 3(a)) and the DC measurements (Fig. 2(a)) is confirmation of the non-dispersive operation observed in small signal measurements (Fig. 2(b)).

The behavior of $\beta$ scales accordingly with observations of the nonlinearity in conductance shown in Fig. 2(a). At low input power, the dependence of $\beta$ on nanowire width is observed to obey a simple exponential scaling law

$$
\beta(w)=\beta_{0} e^{\eta w_{c} / w} .
$$

Here, $\eta$ is a scaling constant, $\beta_{0}$ is an amplitude constant, and $w_{c}=2 r_{s}$ is the critical width. For the $1 \mathrm{GHz}$ data in Fig. 3 (c), an exponential fit yields $\eta=6.15$ and $\beta_{0}=1.1 \mathrm{~V} / \mathrm{W}$ (see the inset of Fig. 3(c)).

For the $100 \mathrm{~nm}$ and $70 \mathrm{~nm}$ GNDs, the responsivity is low near zero bias. As $w$ is larger than a few $w_{c}$ in these structures, the charge neutrality feature is suppressed due to a lack of effective self gating. In the case of the $50 \mathrm{~nm}$ and $30 \mathrm{~nm}$ structures, $w$ approaches $w_{c}$, and an appreciable $\beta$ is observed. As the input power is increased $\beta$ gradually increases as the waveform encounters a gradually decreasing conductance due to velocity saturation in the nanowire. This additional nonlinearity generates a steady rise in $\beta$ at high input power. LSNA measurements are also performed in order to obtain $\beta$ as a function of frequency from $1 \mathrm{GHz}$ to $49 \mathrm{GHz}$ at an input power of $0 \mathrm{dBm}$ (Fig. 3(c)). The associated NEP is calculated by extracting $r_{d}$ from the lowfrequency S-parameter measurements (Fig. 3(d)). A lowfrequency responsivity (NEP) of $250 \mathrm{~V} / \mathrm{W}(50 \mathrm{pW} / \sqrt{\mathrm{Hz}})$ is observed in the $30 \mathrm{~nm}$ GND detector. The GND responsivity is observed to be relatively constant as a function of frequency with the exception of a gradual drop which may be attributed to $C_{p a d}$ and resistive losses in the substrate. Despite the parasitic effect, a flat responsivity (NEP) of $80 \mathrm{~V} / \mathrm{W}(170 \mathrm{pW} / \sqrt{\mathrm{Hz}})$ is observed in the $30 \mathrm{~nm}$ GND detectors at high frequency.

In conclusion, high frequency detection graphene nanowire diodes have been demonstrated. DC, small signal, and large signal measurements are performed in order to investigate the nonlinearities in the device which enable detection. Two sources of nonlinearity are described: lateral gating at low bias and velocity saturation at high bias. A small energy gap may enhance the low bias nonlinearity in narrow graphene nanowires at finite temperature. High frequency measurements are consistent and reveal that the GND detectors obey a simple scaling law. In this work, we establish the feasibility of graphene nanowire diode detectors as a competitive platform for high frequency power detection.

Methods: The GNDs are fabricated in several steps using the JEOL 9300FS EBL system. First, Ohmic contacts are patterned using a liftoff compatible bilayer MMA/ PMMA resist stack. The contact is then formed by deposition of a $\mathrm{Ti} / \mathrm{Pt} / \mathrm{Au}(10 \mathrm{~nm} / 10 \mathrm{~nm} / 70 \mathrm{~nm})$ metal stack. Next, the GND trenches are patterned in PMMA via EBL. Several dose tests were performed in order to determine the optimum dose for achieving the designed nanowire width. The nanowire width was observed to vary within $\pm 3 \mathrm{~nm}$ depending on the dose used in the EBL exposure. As PMMA is positive tone resist, higher doses resulted in channels and wider isolation trenches. A dose of $485 \mu \mathrm{C} / \mathrm{cm}^{2}$ was found to yield device geometry maximally close to the design values. The isolating trenches are then etched using a low pressure ( $3 \mathrm{mTorr} / 50 \mathrm{~W}$ ) $\mathrm{O}_{2}$ plasma for $10 \mathrm{~s}$. This low pressure etch is maximally directional, which is essential for achieving the high edge acuity required for device operation. Following this step, a mesa isolation is achieved via EBL patterning of ma-N 2403 negative tone resist and etching in $(50 \mathrm{mTorr} /$ $50 \mathrm{~W}) \mathrm{O}_{2}$ plasma for $20 \mathrm{~s}$. Finally, Ti/Au(10 nm/100 nm) contact pads are pattered and deposited via EBL patterning of MMA/PMMA and subsequent liftoff. The nanowire width $(w)$ is varied, while the isolation width $\left(w_{c}=100 \mathrm{~nm}\right)$ and channel length $(l=1.1 \mu \mathrm{m})$ are held constant. All devices presented in this work consist of nine parallel GND channels etched into a single mesa. Ancillary structures for characterization included transfer length method (TLM) structures for assessment of the contact resistance and large area sheet 
resistance, and van der Pauw structures for the determination of the low field carrier mobility. Measurements on these structures yielded an average contact resistance of $245 \Omega \mu \mathrm{m}$ and large area sheet resistance of $356 \Omega /$ sq, and room temperature Hall measurements yielded an average mobility(carrier density) of $1400 \mathrm{~cm}^{2} / \mathrm{V} \mathrm{s}\left(1.2 \times 10^{13} \mathrm{~cm}^{-2}\right)$.

This work was supported by the European Science Foundation (ESF) under the EUROCORES Program EuroGRAPHENE, and by the EU Graphene Flagship (No. 604391). We also acknowledge support from the Swedish Foundation for Strategic Research (SSF) and the Knut and Alice Wallenberg Foundation (KAW).

${ }^{1}$ S. Das Sarma, S. Adam, E. H. Hwang, and E. Rossi, Rev. Mod. Phys. 83, 407 (2011).

${ }^{2}$ F. Koppens, T. Mueller, P. Avouris, A. Ferrari, M. Vitiello, and M. Polini, Nat. Nanotechnol. 9, 780 (2014).

${ }^{3}$ A. M. Song, M. Missous, P. Omling, A. R. Peaker, L. Samuelson, and W. Seifert, Appl. Phys. Lett. 83, 1881 (2003).

${ }^{4}$ J. Mateos, B. G. Vasallo, D. Pardo, and T. Gonzlez, Appl. Phys. Lett. 86, 212103 (2005).

${ }^{5}$ C. Balocco, A. M. Song, M. berg, A. Forchel, T. Gonzlez, J. Mateos, I. Maximov, M. Missous, A. A. Rezazadeh, J. Saijets et al., Nano Lett. 5, 1423 (2005).

${ }^{6}$ C. Balocco, S. R. Kasjoo, X. F. Lu, L. Q. Zhang, Y. Alimi, S. Winnerl, and A. M. Song, Appl. Phys. Lett. 98, 223501 (2011).

${ }^{7}$ C. Balocco, S. R. Kasjoo, L. Q. Zhang, Y. Alimi, and A. M. Song, Appl. Phys. Lett. 99, 113511 (2011).

${ }^{8}$ P. Sangaré, G. Ducournau, B. Grimbert, V. Brandli, M. Faucher, C. Gaquire, A. İñiguez-de-la Torre, I. İñiguez-de-la Torre, J. F. Millithaler, J. Mateos et al., J. Appl. Phys. 113, 034305 (2013).

${ }^{9}$ F. Al-Dirini, F. M. Hossain, A. Nirmalathas, and E. Skafidas, Sci. Rep. 4, 3983 (2014).

${ }^{10}$ F. Al-Dirini, F. M. Hossain, A. Nirmalathas, and E. Skafidas, Nanoscale 6, 7628 (2014).

${ }^{11}$ A. Westlund, M. Winters, I. G. Ivanov, J. Hassan, P. Nilsson, E. Janzén, N. Rorsman, and J. Grahn, Appl. Phys. Lett. 106, 093116 (2015).
${ }^{12}$ W. Strupinski, K. Grodecki, A. Wysmolek, R. Stepniewski, T. Szkopek, P. E. Gaskell, A. Gruneis, D. Haberer, R. Bozek, J. Krupka et al., Nano Lett. 11, 1786 (2011).

${ }^{13}$ C. Riedl, C. Coletti, T. Iwasaki, A. A. Zakharov, and U. Starke, Phys. Rev. Lett. 103, 246804 (2009).

${ }^{14}$ C. Riedl, C. Coletti, and U. Starke, J. Phys. D: Appl. Phys. 43, 374009 (2010).

${ }^{15}$ J. A. Robinson, M. Hollander, M. LaBella, K. A. Trumbull, R. Cavalero, and D. W. Snyder, Nano Lett. 11, 3875 (2011).

${ }^{16}$ C. Melios, V. Panchal, C. E. Giusca, W. Strupiński, S. R. P. Silva, and O. Kazakova, Sci. Rep. 5, 10505 (2015).

${ }^{17}$ N. M. R. Peres, A. H. Castro Neto, and F. Guinea, Phys. Rev. B 73, 195411 (2006).

${ }^{18}$ M. Y. Han, B. Özyilmaz, Y. Zhang, and P. Kim, Phys. Rev. Lett. 98, 206805 (2007).

${ }^{19}$ W. J. Yu and X. Duan, Sci. Rep. 3, 1248 (2013).

${ }^{20}$ M. Evaldsson, I. V. Zozoulenko, H. Xu, and T. Heinzel, Phys. Rev. B 78, 161407 (2008).

${ }^{21}$ M. Y. Han, J. C. Brant, and P. Kim, Phys. Rev. Lett. 104, 056801 (2010).

${ }^{22}$ S. Dröscher, P. Roulleau, F. Molitor, P. Studerus, C. Stampfer, K. Ensslin, and T. Ihn, Appl. Phys. Lett. 96, 152104 (2010).

${ }^{23}$ S. Dröscher, P. Roulleau, F. Molitor, P. Studerus, C. Stampfer, K. Ensslin, and T. Ihn, Phys. Scr. 2012, 014009.

${ }^{24}$ M. Winters, E. Ö. Sveinbjörnsson, and N. Rorsman, J. Appl. Phys. 117, 074501 (2015).

${ }^{25}$ See supplementary material at http://dx.doi.org/10.1063/1.4932970 for a derivation of Eq. (1) (2015).

${ }^{26}$ M. M. Fogler, D. S. Novikov, and B. I. Shklovskii, Phys. Rev. B 76, 233402 (2007).

${ }^{27}$ L. Patrick and W. J. Choyke, Phys. Rev. B 2, 2255 (1970).

${ }^{28}$ V. Panchal, A. Lartsev, A. Manzin, R. Yakimova, A. Tzalenchuk, and O. Kazakova, Sci. Rep. 4, 5881 (2014).

${ }^{29}$ M. Winters, J. Hassan, H. Zirath, E. Janzén, and N. Rorsman, J. Appl. Phys. 113, 193708 (2013).

${ }^{30}$ M. Inanc, M. Han, A. Yound, B. Ozyilmaz, P. Kim, and K. Shepard, Nat. Nanotechnol. 3, 654 (2008).

${ }^{31}$ V. E. Dorgan, M.-H. Bae, and E. Pop, Appl. Phys. Lett. 97, 082112 (2010).

${ }^{32}$ H. Nyquist, Phys. Rev. 32, 110 (1928).

${ }^{33} \mathrm{M}$. Thorsell and K. Andersson, IEEE Trans. Microwave Theory Tech. 60, 149 (2012). 